\title{
Reporting research findings to participants is an ethical imperative
}

\author{
Dissemination must become the default expectation for all research
}

Jeremy Taylor independent consultant and writer ${ }^{1}$ former chief executive ${ }^{1}$

${ }^{1} 13$ Pier Head, Wapping High Street, London E1W 1PN, UK; ${ }^{2}$ National Voices

The results of clinical trials should be disseminated to those who took part in them. For a start, it is a basic courtesy, and that should be reason enough. It is also an ethical imperative. The World Medical Association's Declaration of Helsinki insists that "all medical research subjects should be given the option of being informed about the general outcome and results of the study."

Research subjects contribute to the greater good and expose themselves to risk of harm. They have a right to know the outcome of the research. This is especially important for participants with a direct interest in the findings, for whom knowledge can be power.

Reporting back to participants is part of the discipline of transparency that keeps researchers honest and accountable. It fits with the broader responsibility of scientists to communicate their work and foster public understanding. It is part of making patient and public involvement a core strand of health research. It is consistent with a welcome focus on lay summaries, shortly to become mandatory as part of the EU clinical trials directive. ${ }^{2}$ In that context, the results of a recent survey of trial authors by Schroter and colleagues are disappointing. ${ }^{3}$ Fewer than half the respondents had reported (or planned to report) their clinical trial findings to participants. The proportion was similar when it came to sharing results with relevant patient communities. How can we explain this? One clear answer is that researchers are under very little pressure to disseminate results to patients and participants. Respondents in the Schroter study reported that funders, regulators, universities, and ethics committees rarely required (or funded) such dissemination.

In a world where transparency is a widely accepted priority, this is at first surprising. But publication is often taken as a proxy for transparency. Even the AllTrials campaign, ${ }^{4}$ which calls for full disclosure of the methods and results of all clinical trials, has little to say about dissemination. Publication is only the bottom rung of the ladder of transparency. Results might be in the public domain but it doesn't follow that people know about them, can find them, have access to them, or make sense of them.

\section{No excuse}

In the absence of pressure for action, justifications for inaction crowd in. When asked about barriers to dissemination, some respondents to the survey said that people would not be interested in trial results, would not understand them, and might indeed misunderstand them. Similar objections were once made about patients accessing their medical records. These responses reveal untested assumptions and border on the patronising. As with access to medical records, a lack of demand from patients is interpreted as a lack of interest, when it is just as plausibly an indicator that people "don't know what they don't know." Other barriers to dissemination cited in the study cannot be so readily dismissed. There are a host of practical matters, including the logistics of tracking down participants and relevant patient groups, and concerns about unwittingly breaching rules on data protection or the marketing and promotion of pharmaceuticals.

Complex clinical research can be difficult to explain. The results of a trial can have a substantial emotional impact, dashing personal hopes or unwittingly giving false hope. In the wider context, results could be misrepresented, especially in social media. All these are legitimate concerns, but they are not reasons for avoiding communication but rather for doing it well.

Doing it well, however, takes time, will, skill, and resources. It requires researchers to devote thought and energy to targeting individuals and organisations and to tailoring their messages to meet people's varying information needs. Just because something is the right thing to do is no guarantee that it will get done.

Guidance could help, perhaps produced by respected research organisations such as the UK's National Institute for Health Research. It is notable that several of the respondents to the survey reported no problems disseminating results to participants and described successful approaches. Others could learn from their experiences.

But guidance will achieve little without a more fundamental change in culture. Communicating research outcomes to participants and relevant patient and carer communities must 
be the default expectation, built into the design, budgeting, and governance of clinical trials, along with other strands of patient and public involvement.

The BMJ set an excellent example at the start of 2019 by requiring authors of research papers to describe plans for disseminating their findings to participants and other relevant communities, or to declare that they have none. It is time for funders, regulators, institutions, ethics committees, and other journals to follow suit. They should insist that researchers disseminate results to participants and patient communities, and be prepared to consider sanctions for failure to comply.

Commissioned, not peer reviewed.
Competing interests: I have read and understood BMJ policy on declaration of interests and declare the following: I am a member of BMJ's patient panel and a former member of the BMJ editorial board.

1 World Medical Association. Declaration of Helsinki. 2013. www.wma.net/policies-post/ wma-declaration-of-helsinki-ethical-principles-for-medical-research-involving-humansubjects.

2 Expert group on clinical trials for the implementation of Regulation (EU) No 536/2014 on clinical trials. Summaries of clinical trial results for laypersons. 2017. https://ec.europa. eu/health/sites/health/files/files/eudralex/vol-10/2017_01_26_summaries_of_ct_results_ for_laypersons.pdf.

3 Schroter S, Price A, Malički M, Richards T, Clarke M. Frequency and format of clinical trial results dissemination to patients: a survey of authors of trials indexed in PubMed. BMJ Open 2019;9:e032701. 10.1136/bmjopen-2019-03270131636111 4 AllTrials. http://alltrials.net.

Published by the BMJ Publishing Group Limited. For permission to use (where not already granted under a licence) please go to http://group.bmj.com/group/rights-licensing/ permissions 